\title{
Fracturas Mandibulares Conminutadas por Impacto de Proyectil Balístico: Propuesta de Tratamiento Inicial Mediante Método de Fijación Alternativo
}

\author{
Mandibular Fractures Comminuted by Ballistic Projectile Impact: \\ Initial Treatment Proposal Using Alternative Fixation Method
}

\author{
Marcelo Mardones Muñoz ${ }^{1,2}$; Rodrigo Bravo Ahumada ${ }^{1,2}$; Jack Altschiller Mardones ${ }^{1}$ \& Hernán Pérez Gutiérrez ${ }^{3}$
}

MARDONES, M. M.; BRAVO, A. R.; ALTSCHILLER, M. J. \& PÉREZ, G. H. Fracturas mandibulares conminutadas por Impacto de proyectil balístico: Propuesta de tratamiento inicial mediante método de fijación alternativo. Int. J. Odontostomat., 12(4):423-430, 2018.

RESUMEN: El trauma maxilofacial por arma de fuego representa un desafío terapéutico principalmente debido a la gran conminución de tejidos que genera. Específicamente, en casos de fracturas mandibulares conminutadas, las modalidades de tratamientos incluye la reducción cerrada, fijación con tutor externo, fijación interna con alambres y reducción abierta y fijación interna utilizando placas y tornillos. El objetivo del presente trabajo es describir un tratamiento inicial con un método de fijación para fracturas mandibulares conminutadas como dispositivo alternativo al tutor externo clásicamente utilizado. Se desarrolla una revisión de la literatura actual acerca del tratamiento inicial o de urgencia de fracturas mandibulares conminutadas por impacto de proyectil balístico, haciendo énfasis en las indicaciones de cada modalidad de tratamiento y sus respectivas ventajas y desventajas. Luego se describe detalladamente el proceso de confección de un método de fijación alternativo junto con la exposición de casos clínicos donde fue utilizado como alternativa de tratamiento. La reducción y fijación obtenida por el dispositivo presentado logra resultados satisfactorios, sin encontrarse diferencias significativas a los elementos de fijación clásicos. Tanto el uso de placas de reconstrucción como el de tutor externo constituyen alternativas válidas para el tratamiento de fracturas mandibulares conminutadas. El dispositivo descrito y utilizado en los pacientes es una alternativa eficiente, de fácil confección y bajo costo económico demostrando buenos resultados en relación a la consolidación de fracturas conminutadas. Se debe conocer las indicaciones para la aplicación de cada modalidad de tratamiento.

PALABRAS CLAVE: fractura mandibular, conminución, proyectil balístico, placa de reconstrucción, tutor externo.

\section{INTRODUCCIÓN}

Las fracturas mandibulares conminutadas son complejas y generalmente son el resultado de un impacto significativo en un área localizada de este hueso ya sea por una colisión a gran velocidad o por un impacto de proyectil. Específicamente, el trauma maxilofacial por arma de fuego representa un desafío terapéutico principalmente debido a la gran conminución que se genera. En casos de fracturas mandibulares conminutadas, las modalidades de tratamientos incluye la reducción cerrada, fijación con tutor externo, y reducción abierta y fijación interna utilizando placas y tornillos. Algunos de los factores que van a determinar la alternativa terapéutica a utilizar son la ubicación de la fractura, grado de conminución, comfort para el paciente, experiencia del equipo tratante y factores socioeconómicos. (Bede et al., 2017; Palacios Vivar et al., 2017).

El manejo de las fracturas mandibulares conminutadas ha evolucionado. Tradicionalmente, nunca se accedía a la fractura para así evitar la desvitalización de los fragmentos óseos que finalmente se podían perder, infectar o secuestrar. Salvo algunas excepciones, estos casos eran manejados con técni-

\footnotetext{
${ }^{1}$ Equipo de Cirugía Maxilofacial Hospital San José, Independencia, Santiago, Chile.

${ }^{2}$ Facultad de Odontología, Universidad de Chile, Santiago, Chile.

${ }^{3}$ Cirujano Maxilofacial, Santiago, Chile.
} 
cas cerradas basadas en la fijación intermaxilar, férulas o ambos. El manejo clásico de una herida por arma de fuego de mandíbula, seguido del manejo de la vía aérea, es descartar una lesión vascular (Chrcanovic et al., 2013). Luego, el aseo quirúrgico tanto del tejido blando como duro dependía del tipo de herida pero generalmente involucraba la remoción de fragmentos óseos que pudiesen estar en el tejido blando teniendo cuidado de no comprometer la integridad del periosteo de aquellos fragmentos vitales. Cualquier fragmento de hueso con tejido blando adherido a él se consideraba potencialmente viable. Finalmente se extraían los restos de tejido dentario y aquellos dientes con mal pronóstico asociados a la fractura y luego el cierre del tejido blando. El resto del tratamiento estaba basado en la fijación intermaxilar o uso de tutores externos para asegurar la consolidación de los fragmentos conminutados. De manera lógica, este tipo de manejo y tratamiento era prolongado, con tiempos de tratamientos de meses o incluso años en algunos casos. Sin embargo fue considerado el gold estándar durante mucho tiempo (Quintana Díaz et al., 2015).

La introducción de la fijación interna rígida, que evidentemente ha acortado de manera dramática los tiempos de tratamiento, lleva al debate acerca de que modalidad de tratamiento es preferible en casos de fracturas mandibulares conminutadas. Existen reportes acerca del uso de este tipo de tratamiento en Europa ya en los años 70 y su aplicación se fundamentaba en minimizar el secuestro óseo y permitir la función post operatoria de forma temprana. Esencialmente, una reducción abierta y fijación interna de la fractura conminutada completa se realiza usando material de osteosíntesis de carga soportada y si es necesario cualquier defecto óseo puede ser injertado. En este protocolo en particular, la placa debe ser del grosor y perfil suficiente para poder soportar las fuerzas en esta área de la mandíbula. La estabilización por compresión o cualquier otra forma de osteosíntesis de carga compartida esta obviamente contraindicada ya que los pequeños fragmentos de hueso no pueden ser comprimidos y no son capaces de compartir la carga (Alpert et al., 2009; Wojcik et al., 2016).

El tratamiento comienza con el bloqueo intermaxilar en oclusión estable lo cual se logra con arcos y alambres y estabiliza tanto los dientes como el segmento alveolar. Cuando se expone la fractura, generalmente por un acceso extraoral, se debe intentar mantener el periosteo lingual en la medida de lo posible. Los fragmentos pequeños son unidos entre ellos con miniplacas o tornillos tipo "lag screws" en lo que se conoce como simplificación de la fractura. Los segmentos simplificados son luego conectados con una placa de reconstrucción bloqueada con tres o cuatro tornillos en cada extremo de la fractura (Chrcanovic et al.). La mayor cantidad de experiencia predecible se ha obtenido utilizando placas de reconstrucción de sistemas $2,7 \mathrm{~mm}$, placas bloqueadas de $2,4 \mathrm{~mm}$ y últimamente se sabe acerca del uso de placas bloqueadas de 2,0 mm de perfil ancho aunque aún no está comprobado del todo si éstas últimas ofrecen la fuerza y estabilidad suficiente. Finalmente, se desbloquea la oclusión permitiendo al menos una función limitada para el paciente (Rana et al., 2014).

Si bien el tratamiento conservador en base al uso de tutores externos fue una de las primeras modalidades terapéuticas de las fracturas mandibulares conminutadas y aun con la introducción de materiales de osteosíntesis de fijación interna, éstos continúan vigentes con sus respectivas indicaciones y ventajas. Los tutores externos mandibulares son unas de las herramientas que los cirujanos maxilofaciales y traumatólogos tienen para tratar fracturas complejas conminutadas, especialmente cuando se trata de trauma por impacto de proyectil balístico (Chao et al., 2017; Palacios Vivar et al., 2017).

En el trabajo, presentamos un tutor externo alternativo, confeccionado a mano en el tiempo intraoperatorio. Tiene las ventajas de ser de un bajo costo económico, fácil de fabricar e implementar y su eficiencia lo hace bastante útil hasta el día de hoy. Esta técnica puede ser una alternativa válida y útil al tutor externo convencional.

\section{MATERIAL Y MÉTODO}

Los tutores externos se utilizan como tratamiento inicial de control de daño en fracturas mandibulares conminutadas, especialmente en pacientes con déficit de tejidos blandos, para estabilizar fragmentos óseos, en ese contexto es primordial asegurar la vía aérea y por lo general los pacientes son intubados precozmente quedando en primera instancia con ventilación asistida, hasta asegurarse una correcta estabilización sin riesgo de compromiso de vía aérea.

Una vez resguardada la vía aérea, lo primero es realizar adecuada hemostasia y aseo quirúrgico de la zona para luego continuar con manejo de tejidos blandos en que es fundamental la unión de los tejidos 
remanentes asegurando una adecuada vascularización, no traccionando ni comprimiendo los bordes de la herida y eliminando los tejidos desvitalizados. Las heridas deben tener un seguimiento acucioso, con aseo diario y evaluando signos de desvitalización o infección, el retiro de sutura debe realizarse entre 5 a 7 días en piel.

En caso de heridas por proyectil balístico es fundamental la obtención de imágenes, en este caso el gold estándar es la Tomografía Computarizada (TC) Craneofacial. Por lo general el momento ideal es previo a la reconstrucción de tejidos blandos, pero dadas las circunstancias en que prima la vía aérea y la hemostasia, se toma la TC posterior a esto, pudiendo recién dimensionar el daño a tejidos óseos, la ubicación del proyectily con eso poder planificar la mejor manera de estabilizar segmentos o reconstruir adecuadamente.

Es de rigor chequear los antecedentes médicos del paciente y evaluar si es necesario la aplicación de vacuna antitetánica.

Al ser una herida que compromete tanto piel como cavidad bucal es necesario utilizar antibióticos vía endovenosa de amplio espectro con inhibidores de la betalactamasa, como Amoxicilina + ácido clavulánico o Ampicilina + Sulbactam (este último fue el utilizado en nuestro centro hospitalario por disponibilidad, costos y aprobación del comité de infectología, evaluándose en conjunto a este último el tiempo de administración según evolución).

Para la confección del tutor externo, que proponemos, se requieren los siguientes materiales:

- Agujas Kirschner de 2.0 (de preferencia con hilo en la punta y mínimo 3 por cada segmento)

- Taladro Percutor con batería portátil (que pueda ser esterilizado)

- Tubo de silicona (Se utilizó manguera de silicona para aspiración de 2 milímetros de espesor, con un diámetro interno de $7 \mathrm{~mm}$ y $11 \mathrm{~mm}$ de diámetro total)

- Polvo y líquido de Acrílico de autopolimerización

- Frasco y espátula para preparación de acrílico

- Jeringa de 60 cc

- Cizalla cortante de acero

Para la instalación del tutor externo, el paciente debe estar bajo anestesia general. Se realiza una pequeña incisión en piel a nivel de donde se desea instalar la aguja Kirschner, se libera el tejido hasta llegar al plano óseo y se procede a la instalación de la aguja con el taladro percutor teniendo precaución de estructuras nobles, perforando de forma bicortical el hueso mandibular, para lograr estabilidad. Se instala mínimo 3 agujas kirschner por segmento idealmente. Luego se adapta el tubo de silicona con un largo y forma adecuada, con pequeños cortes en este se logra que sea atravesado por las agujas de Kirschner. Se prepara acrílico de autopolimerización en forma fluida y rápidamente se vacía en una jeringa de 60 cc para con esta traspasar al tubo de silicona en toda su extensión. Se mantiene el tubo en posición hasta que polimerice por completo el acrílico, teniendo precaución de quitar los excesos y enfriar con compresas húmedas o suero el tubo ya que el acrílico al polimerizar produce una reacción exotérmica. Una vez solidificado el acrílico se procede a cortar los excesos sobresalientes de las agujas con la cizalla de acero.

El paciente debe mantenerse en aseos constantes, chequeando que no haya infección en las zonas donde quedaron instaladas las agujas. El tutor externo estabiliza los segmentos y se rige bajo la fisiología de reparación ósea por lo que permanece al menos 1 mes en posición para lograr consolidación ósea, idealmente 2 meses para obtener remodelación ósea.

Retirar el tutor es bastante sencillo e incluso se podría realizar con anestesia local; se corta el tutor en varios segmentos con una sierra o disco de carborundum y se procede a retirar las agujas con un instrumento como alicate de alambre quirúrgico o porta alambre, lavando con suero fisiológico las zonas de perforación y suturando a nivel cutáneo.

\section{RESULTADOS}

A continuación se presentan 4 casos de fracturas mandibulares tratadas mediante el uso de tutor externo.

\section{Caso Clínico 1}

Paciente de sexo masculino, 24 años de edad, con diagnóstico de fractura conminutada mandibular izquierda por impacto de proyectil balístico.

Se observa gran conminución del cuerpo mandibular izquierdo (Figs. 1 y 2) con un fragmento central de mayor tamaño. Mediante el uso de un tutor externo fabricado de manera intra operatoria, fijado 


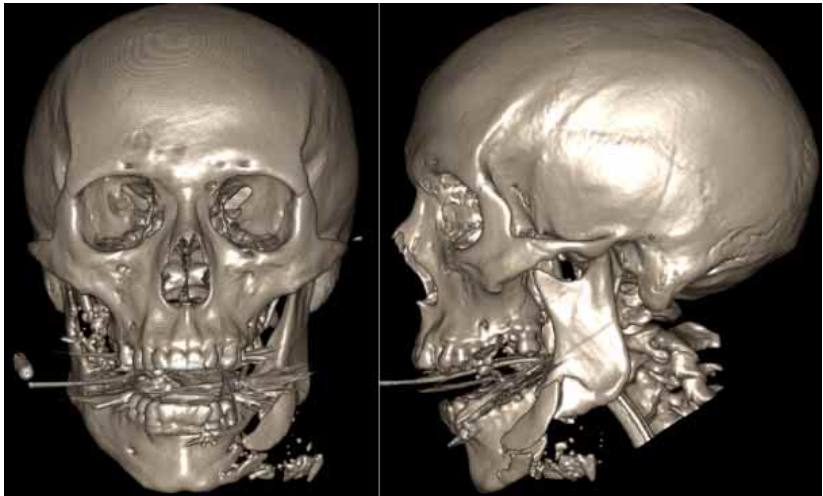

Fig. 1. CT reconstrucción 3D, pre quirúrgico.

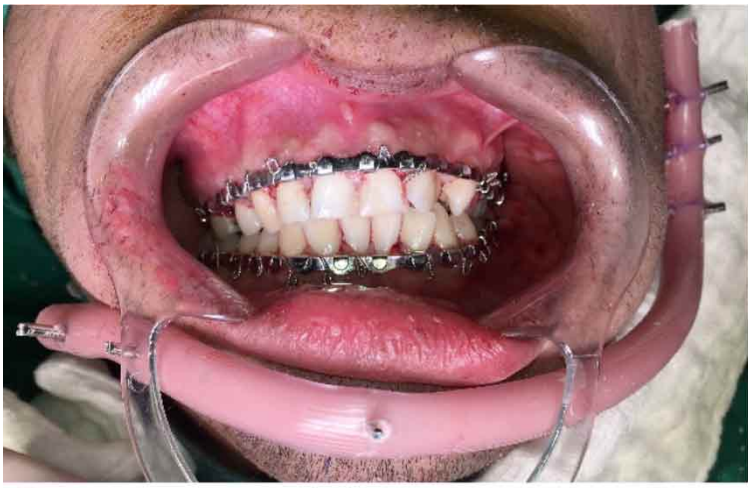

Fig. 3. Fotografía clínica, post operatorio inmediato

con 3 tornillos en el lado izquierdo y 2 tornillos en el lado derecho, se logra una oclusión estable (Figs. 3 y 4.).

\section{Caso Clínico 2}

Paciente de sexo masculino, 21 años de edad, sufre impacto balístico de gran intensidad en el cuerpo mandibular derecho, provocando una fractura

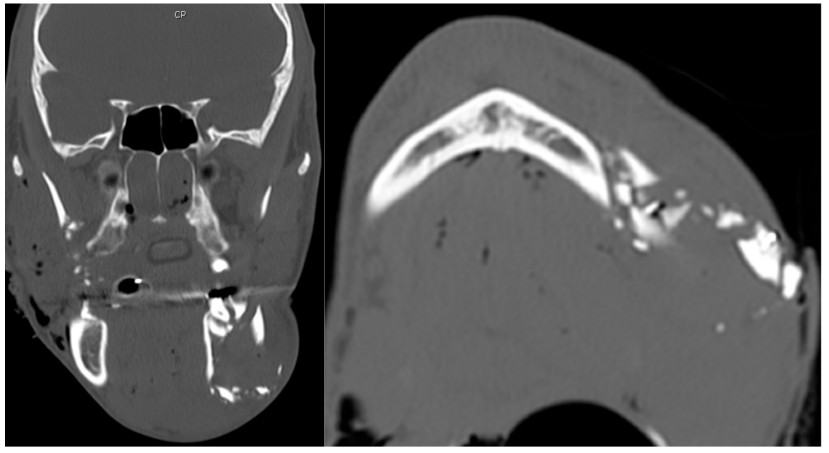

Fig. 2. CT corte coronal y axial, pre quirúrgico

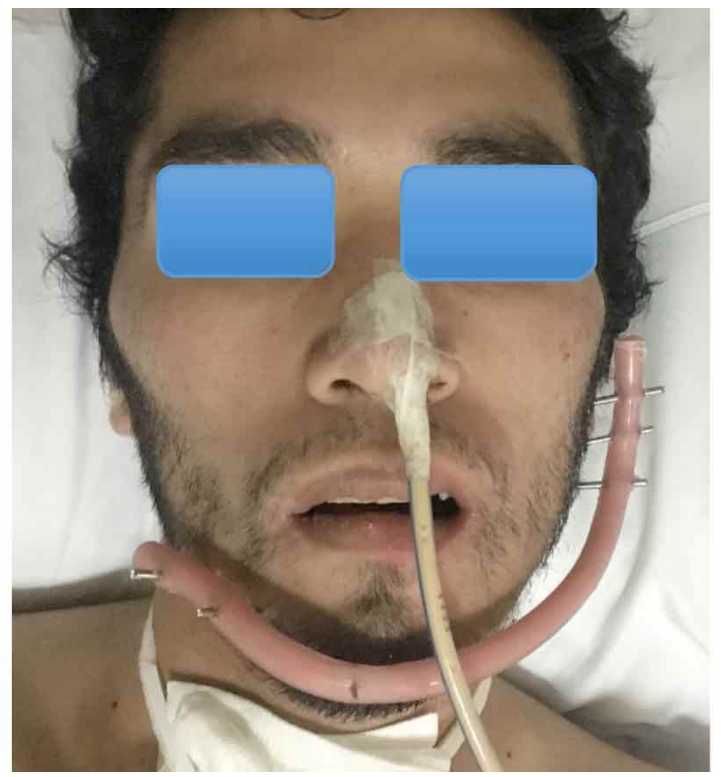

conminutada de alta complejidad, pérdida de tejidos blandos y evidente contaminación de la zona con restos del proyectil (Figs. 5 a 7 ).

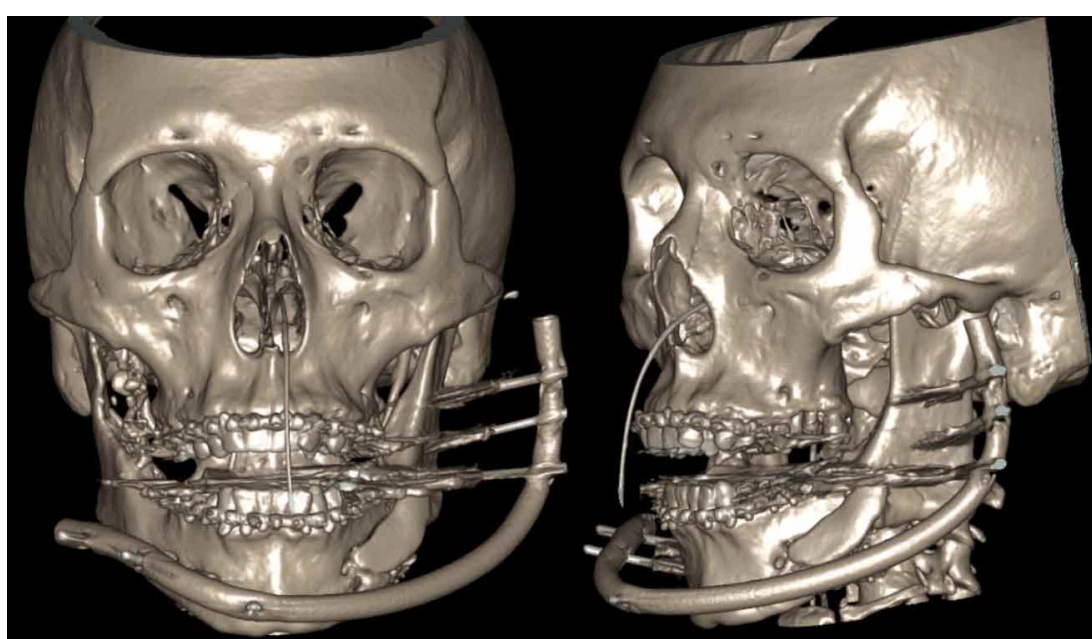

Fig. 4. CT reconstrucción 3D de control post quirúrgico.

\section{Caso Clínico 3}

Paciente de sexo masculino, 19 de años de edad, sufre impacto de proyectil balístico en región mandibular anterior y posterior derecha, provocando gran conminución ósea y alteración de tejido blando (Figs. 8 a 11).

\section{Caso Clínico 4}

Paciente de sexo masculino, 53 años de edad, con diagnóstico de osteomielitis mandibular, se le 


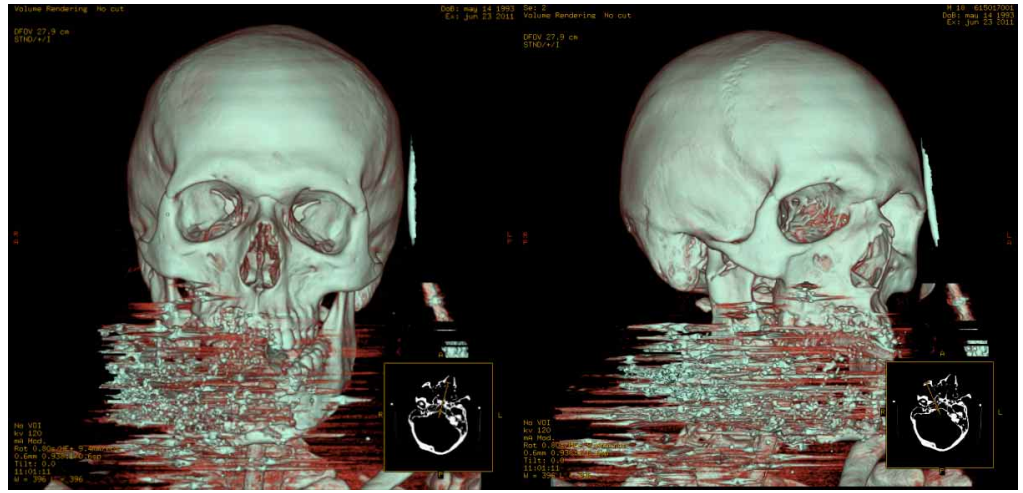

Fig. 5. CT reconstrucción 3D, pre quirúrgico.

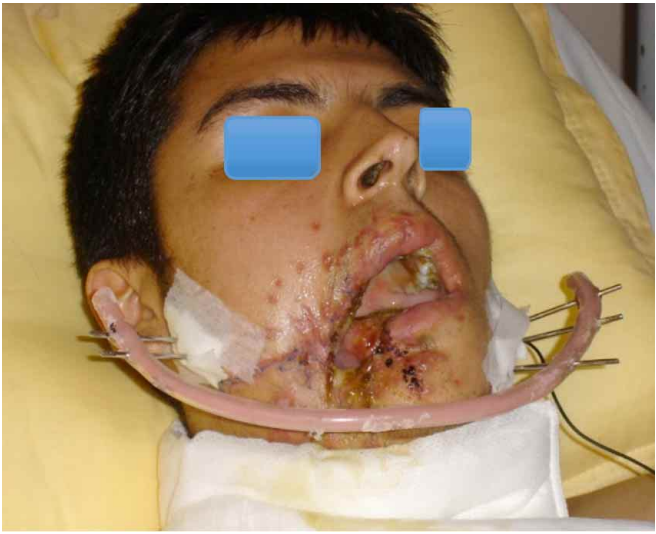

Fig. 6. Fotografía clínica, post operatorio inmediato.

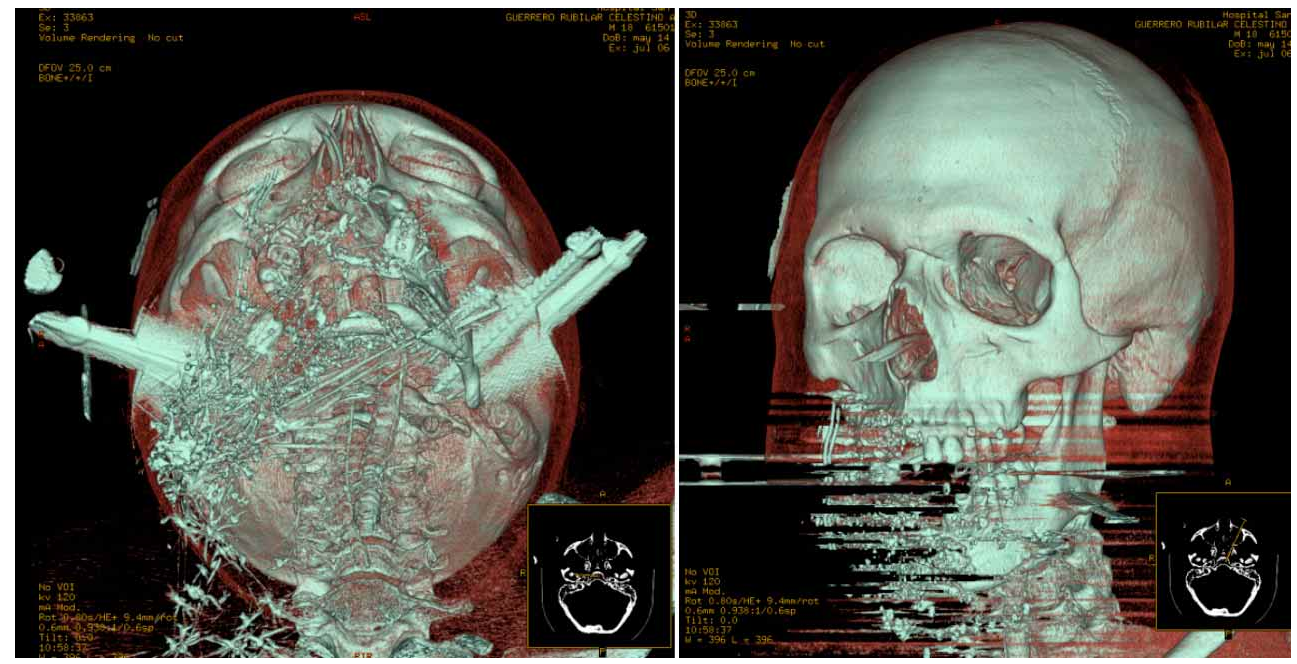

Fig. 7. CT reconstrucción 3D de control post quirúrgico.

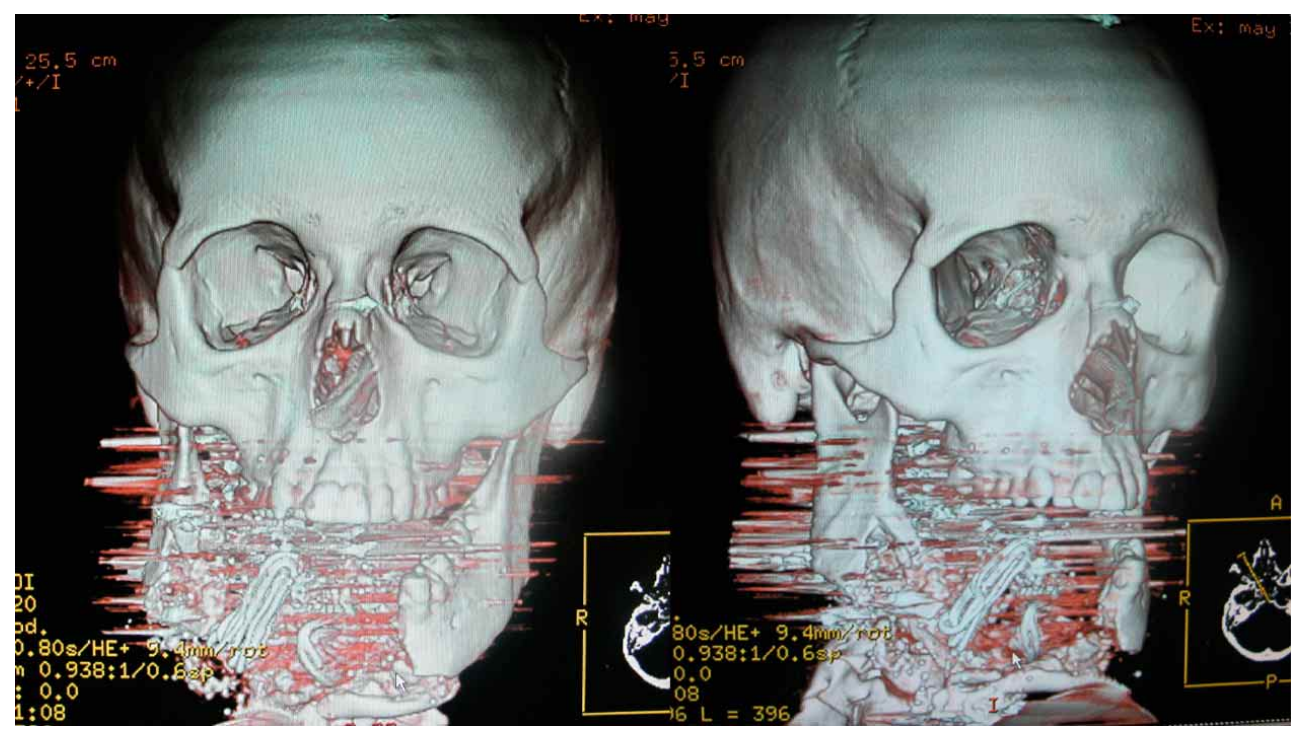

Fig. 8. CT reconstrucción 3D, pre quirúrgico. 

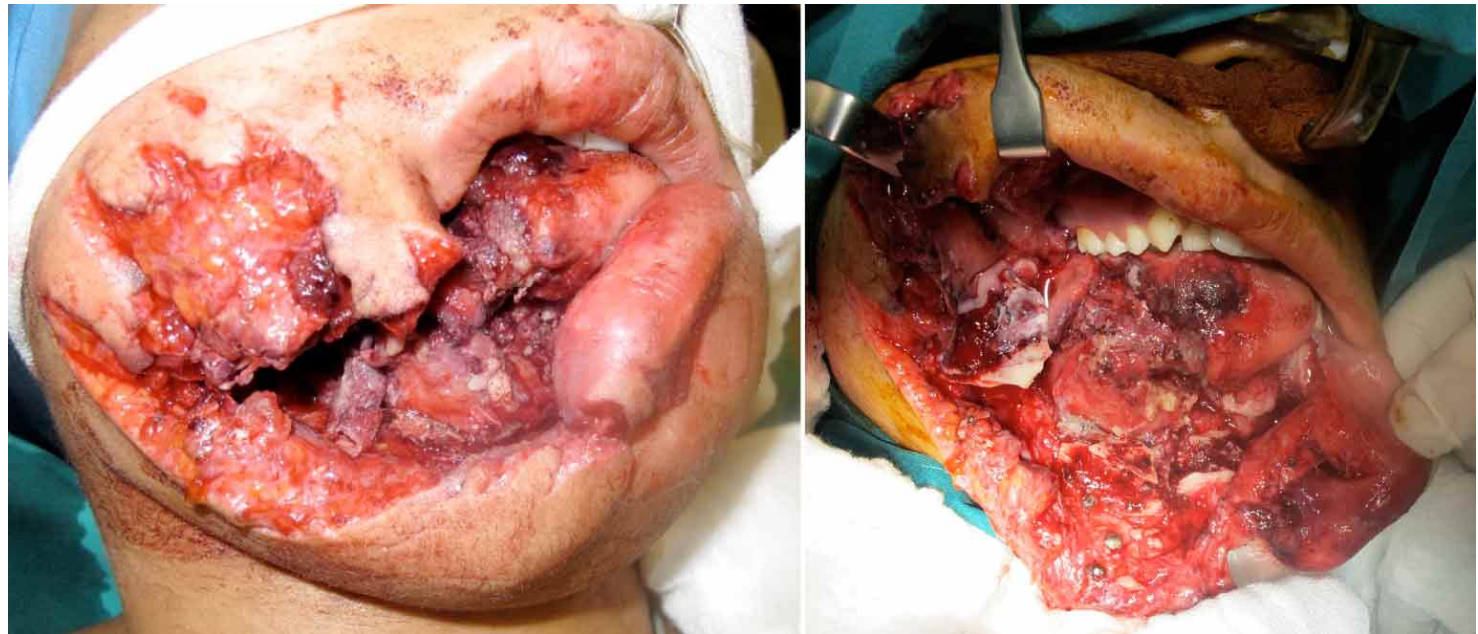

Fig. 9. Fotografía clínica, intra operatorio.

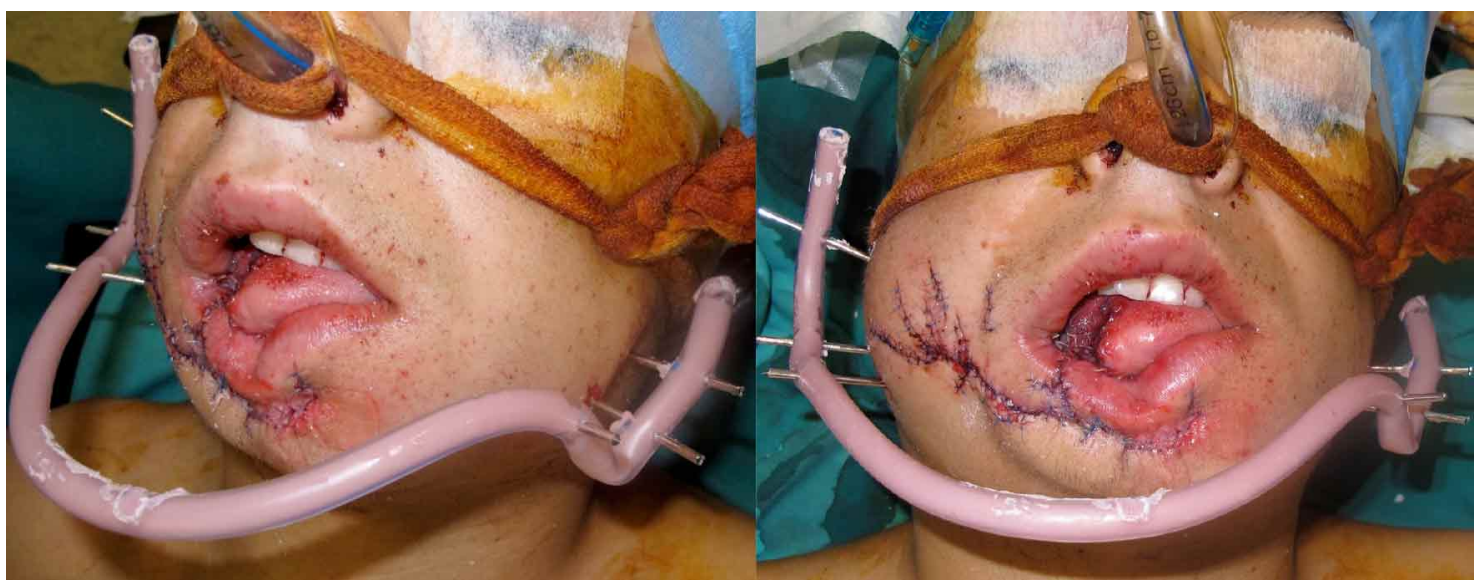

Fig. 10. Fotografía clínica, post operatorio inmediato.

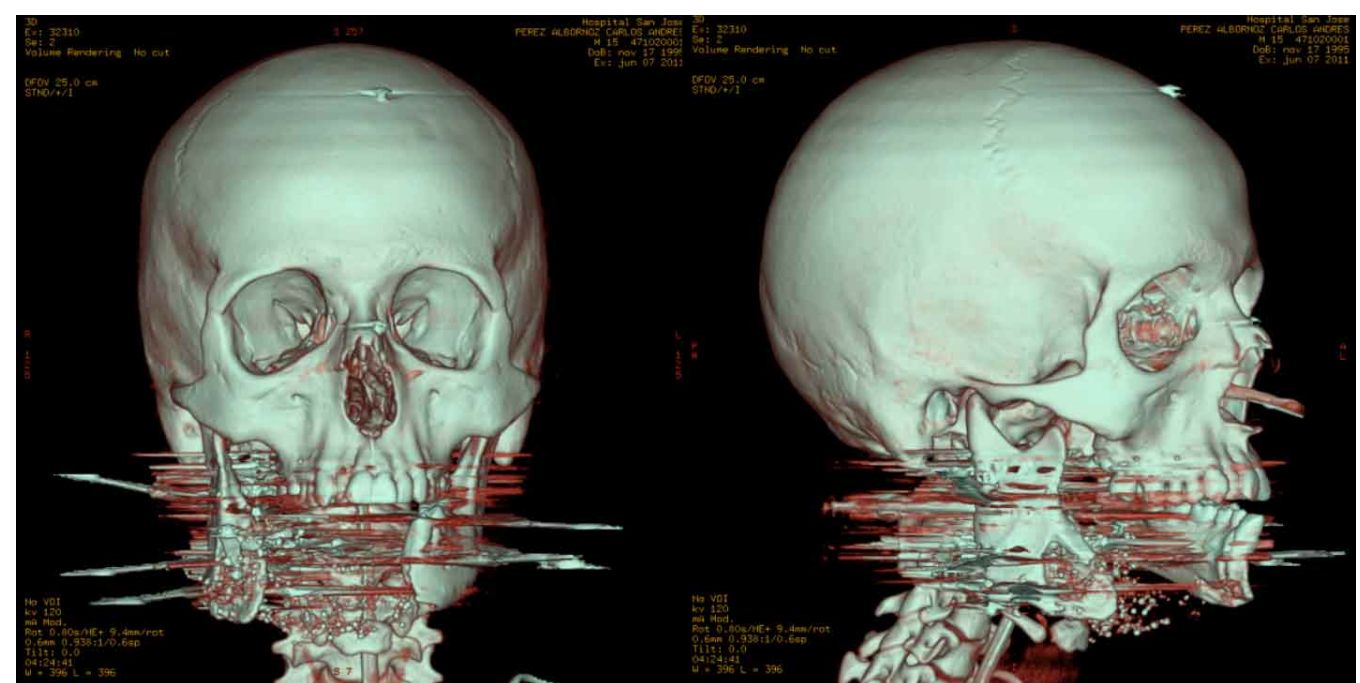

Fig. 11. CT reconstrucción 3D de control post quirúrgico.

realiza resección ósea. Si bien este caso no representa una fractura conminuta, fue tratado con tutor externo para estabilizar segmentos remanentes hasta la cirugía reconstructiva (Figs. 12 a 14). 

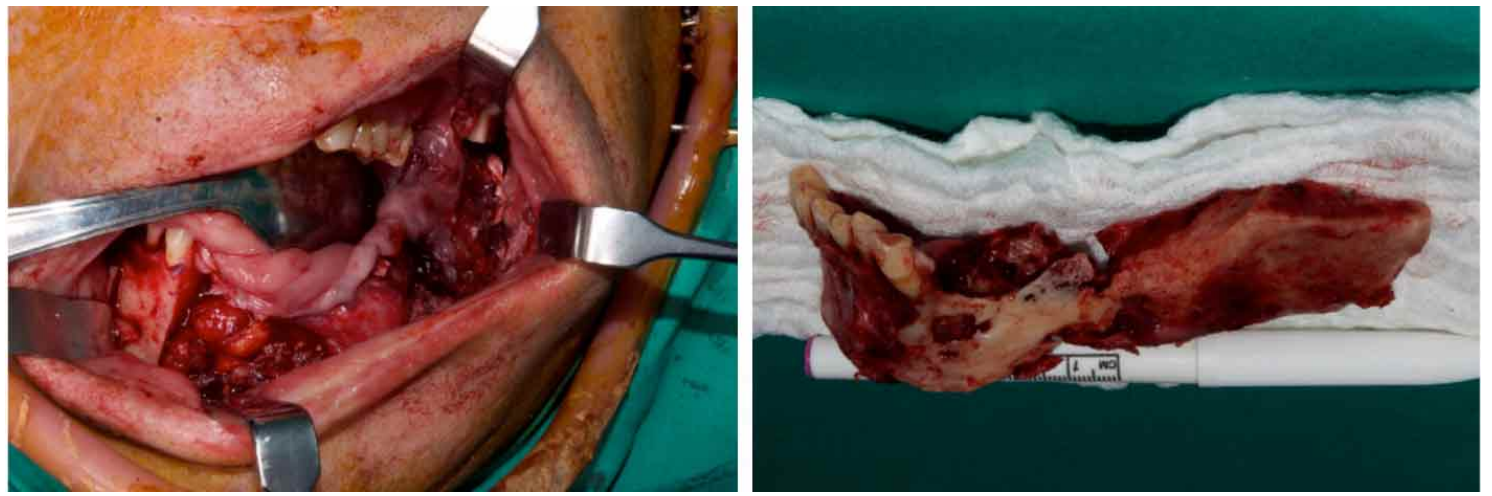

Fig. 12. Fotografía clínica de resección mandibular por osteomielitis.

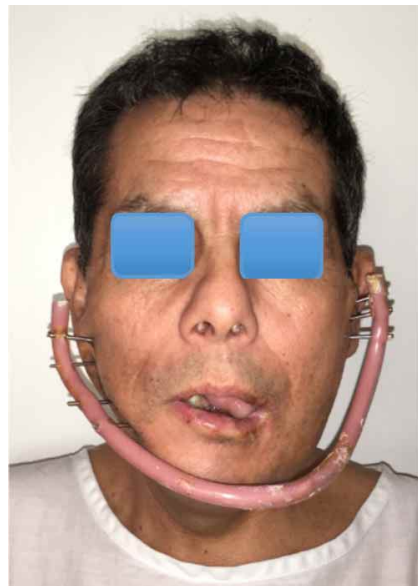

Fig. 13. Fotografía clínica de tutor externo instalado.
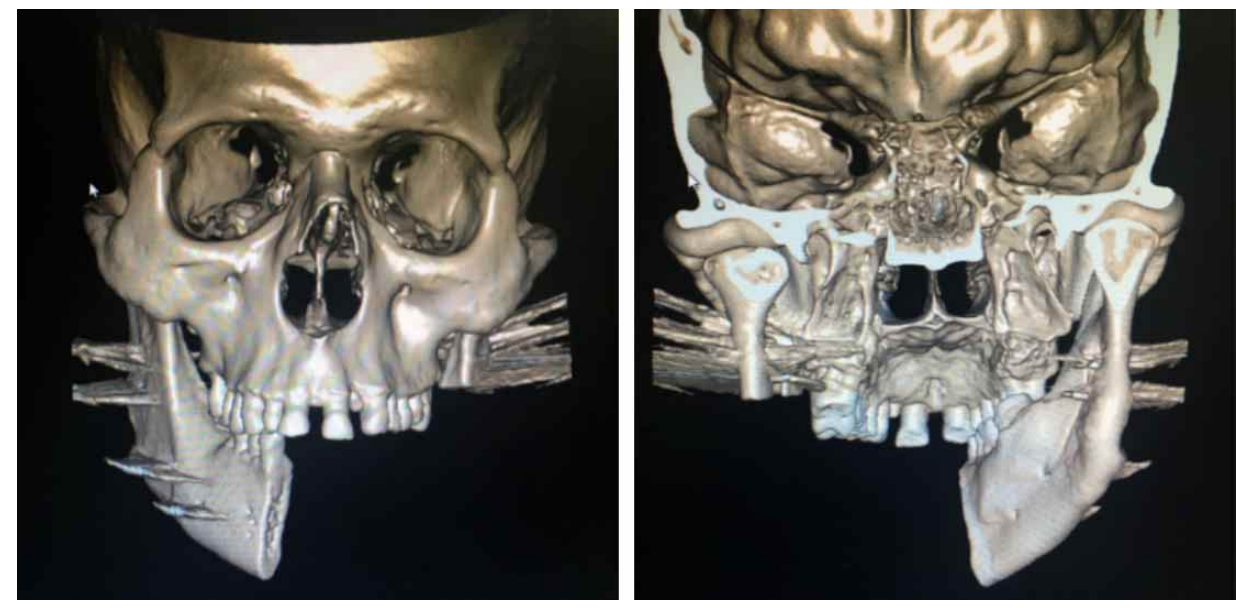

Fig. 14. CT control post quirúrgico, instalación de tutor externo.

\section{DISCUSIÓN}

El trauma maxilofacial por proyectil balístico representa un evidente desafío para el cirujano, principalmente por el gran nivel de conminución provocado. En casos de fractura mandibular conminutada, la literatura actual disponible es clara en definir qué alternativas de tratamiento son las más utilizadas, con mejores ventajas en cuanto a la consolidación de la fractura y entregándole el mejor pronóstico al paciente.

Es conocido que el gold estándar para el tratamiento de este tipo de fracturas es la fijación externa mediante placas y tornillos, específicamente placas de reconstrucción. Alpert et al. y Rana et al. hacen énfasis en que toda fractura mandibular conminutada debiese ser tratada mediante placas y tornillos, principalmente por el excelente confort entregado al paciente, rápida reinserción social y buenos resultados en cuanto a la consolidación ósea. Del mismo modo, Schenkel et al. (2014) propone el uso de placa de re- construcción para el tratamiento de este tipo de fractura, sin embargo en casos de conminución extrema y compromiso de tejido blando, propone el uso de fijación externa. Las ventajas que posee la fijación externa apuntan principalmente a no desperiostizar la zona conminutada asegurando que todos los fragmentos óseos queden contenidos bajo la capa de periosteo sin riesgo de sufrir secuestros o necrosis. Es por eso, que diversos autores defienden el uso de tutores externos para el tratamiento, incluso, definitivo para las fracturas mandibulares conminutadas, a pesar de ciertas desventajas que pudiesen tener, como por ejemplo, menor confort para el paciente y tiempos de tratamiento más largos. Chao et al. apunta a que toda fractura mandibular conminutada debiese ser, por lo menos, inicialmente tratado mediante fijación externa para asegurar la consolidación de todos los fragmentos óseos, más aun cuando existe pérdida de tejido blando que complicaría la utilización de placas y tornillos. 
El tutor externo alternativo presentado tiene la ventaja de cumplir a la perfección el objetivo principal de un tutor convencional, fijando de forma estable los segmentos óseos. El costo de este tutor presentado es menor en relación a los convencionales, ya que se puede realizar de forma artesanal con materiales de uso común en traumatología y odontología. Otra ventaja es que su extensión o tamaño puede fácilmente adaptarse a las necesidades del paciente y condición clínica, ya que se realiza en el mismo acto operatorio y los materiales ocupados permiten confeccionar el diseño a cada caso en particular.

\section{CONCLUSIÓN}

Tanto la fijación interna mediante placas y tornillos como la fijación externa mediante el uso de tutores son alternativas válidas para el tratamiento de fracturas mandibulares conminutadas por impacto de proyectil balístico. La utilización de tutor externo demostró resultados satisfactorios para el tratamiento de este tipo de fractura. El tutor externo alternativo presentado, tiene una funcionalidad idéntica a los tutores convencionales, presentado ventajas respecto al costo, adaptación y extensión según los requerimientos clínicos. Se debe conocer la indicación, ventaja y desventaja de cada modalidad de tratamiento y abarcar cada caso de manera particular tomando en cuenta el contexto socioeconómico del paciente y experiencia del equipo quirúrgico.

MARDONES, M. M.; BRAVO, A. R.; ALTSCHILLER, M. J.. \& PÉREZ, G. H. Mandibular fractures comminuted by ballistic projectile impact: Initial treatment proposal using alternative fixation method. Int. J. Odontostomat., 12(4):423-430, 2018.

ABSTRACT: Ballistic maxillofacial trauma represents a challenge for surgeons because of the important comminution process it presents. Specifically, in cases of mandibular comminuted fractures, the different treatment modalities include closed reduction, external fixation and internal fixation. The purpose of this case series is to describe an initial treatment modality, with a fixation method for comminuted mandibular fractures as an alternative for the classic external fixator. We conducted a literature review about the initial treatment for ballistic comminuted mandibular fractures, emphasizing indications of each treatment modality and their respective advantages and disadvantages. Next, we described the preparation process of an alternative fixation method along with a case series, where it was used as a therapeutical alternative. The reduction as well the fixation achieved by the external fixator, showed satisfactory results without significant differences noted with traditional fixation methods. Both, reconstruction plates and external fixators, are valid alternatives for treating comminuted mandibular fractures. The method described and used in these patients is and efficient alternative, easy to make and at a low cost, with good results in relation to fracture consolidation. We propose that every treatment modality and its indications should be evaluated in order to treat each case adequately.

KEY WORDS: mandibular fracture, comminution, ballistic projectile, reconstruction plate, external fixator.

\section{REFERENCIAS BIBLIOGRÁFICAS}

Alpert, B.; Tiwana, P. S. \& Kushner, G. M. Management of comminuted fractures of the mandible. Oral Maxillofac. Surg. Clin. North Am., 21(2):185-92, 2009.

Bede, S. Y. H.; Ismael, W. K. \& Al-Assaf, D. Characteristics of mandibular injuries caused by bullets and improvised explosive devices: a comparative study. Int. J. Oral Maxillofac. Surg., 46(10):1271-5, 2017.

Chao, D. M.; Jin, S.; Khatri, K. S.; Long, Z. J.; Lei, T. \& Lisheng, H. Managing a grossly comminuted and infected mandibular fracture using a maxillary extra-oral distractor as stabilizing agent: A clinical case report. Trauma Case Rep., 9:10-2, 2017.

Chrcanovic, B. R. Open versus closed reduction: comminuted mandibular fractures. Oral Maxillofac. Surg., 17(2):95-104, 2013.

Palacios Vivar, D. E.; Miranda Villasana, J. E. \& Calderón Lumbreras, A. S. Herida facial por proyectil de arma de fuego: revisión de literatura y estudio clínico de tres casos. Rev. Odontol. Mex., 21(2):12734, 2017.

Quintana Díaz, J. C.; Villareal Corbo, N. \& Quintana Giralt, M. Fractura mandibular por herida de arma de fuego: Presentación de un caso. Acta Odontol. Colomb., 5(1):133-8, 2015.

Rana, M.; Warraich, R.; Rashad, A.; von See, C.; Channar, K. A.; Rana, M.; Stoetzer, M. \& Gellrich, N. C. Management of comminuted but continuous mandible defects after gunshot injuries. Injury, 45(1):20611, 2014.

Schenkel, J. S.; Obwegeser, J.; Zemann, W.; Rostetter, C.; Tandon, R. \& Metzler, P. Outcome of comminuted mandibular fracture repair using an intraoral approach for osteosynthesis. J. Craniofac. Surg., 25(6):2033-7, 2014.

Wojcik, T.; Nicot, R.; Ferri, J. \& Raoul, G. A cheap hand-made mandibular external fixator? J. Craniofac. Surg., 27(7):1839-41, 2016.

Dirección para correspondencia:

Jack Altschiller Mardones

Hospital San José

Independencia

Santiago

CHILE

Email: jaltschillerm@gmail.com

Recibido : 10-07-2018

Aceptado: 31-08-2018 